\title{
ESTUDIO DEL COMPORTAMIENTO FRENTE A CICLOS HIELO- DESHIELO DE MORTEROS DE CEMENTOS DE ACTIVACIÓN ALCALINA OBTENIDOS A PARTIR DE RESIDUOS CERÁMICOS
}

\author{
J. COSA, V. GENOVÉS, L. SORIANO, J. PAYÁ, M. V. BORRACHERO, J. M. MONZÓ \\ Instituto de Ciencia y Tecnología del Hormigón, Universitat Politècnica de València, València, España.
}

\begin{abstract}
RESUMEN
Los Objetivos de Desarrollo Sostenible promulgados por Naciones Unidas promueven la necesidad de reducir la huella de carbono para mitigar el cambio climático. Los cementos de activación alcalina se presentan como una alternativa más sostenible frente al cemento portland cuya huella de carbono es muy elevada. Se prepararon morteros de activación alcalina utilizando un residuo de cerámica sanitaria y el residuo de catalizador de craqueo catalítico. Dichos morteros se sometieron a un número creciente de ciclos hielo-deshielo, estudiando, mediante ensayos no destructivos y resistencia mecánica, el daño sufrido por los mismos y se compararon con los obtenidos por un mortero tradicional fabricado con cemento portland. Los resultados obtenidos hasta 70 ciclos H-D muestran un buen comportamiento de las matrices activadas alcalinamente.

Palabras clave: Índice de Chuva Dirigida; Durabilidade de Fachadas; Mapa do Paraná; Variação Climatológica.
\end{abstract}

\begin{abstract}
The Sustainable Development Goals promulgated by the United Nations promote the need to reduce the carbon footprint to mitigate climate change. The alkali activated cements are presented as a more sustainable alternative material than the Portland cement. Alkali activated mortars were prepared by using sanitary ceramic waste and the catalytic cracking catalyst residue. The mortars were submitted to freezing-thawing cycles. The damage produced is studied by non-destructive tests and mechanical resistance. The results are compared with traditional mortars with Portland cement. The results obtained up to $70 \mathrm{H}-\mathrm{D}$ cycles showed a good behaviour of the alkali activated mixtures.
\end{abstract}

Keywords: wastes, alkali activation, durability, non-destructive test.

\section{RESUMO}

Os Objetivos do Desenvolvimento Sustentável anunciados pelas Nações Unidas mostram a necessidade de reduzir as emissões de carbono para minimizar a mudança climática. Os cimentos ativados alcalinamente se apresentam como uma alternativa mais sustentável em comparação ao cimento Portland cuja emissão de carbono associada é bastante elevada. Foram preparadas argamassas ativadas alcalinamente utilizando resíduo cerâmico e o resíduo de catalizador de craqueamento catalítico. As argamassas foram submetidas a números crescentes de ciclos de gelo-degelo, estudando, através de ensaios não-destrutivos e de resistência mecânica o dano gerado e os resultados foram comparados com uma argamassa tradicional produzida com cimento Portland. Os resultados obtidos até 70 ciclos de gelo-degelo mostram um bom comportamento das matrizes ativadas alcalinamente.

Palavras-chave: resíduos; ativação alcalina; durabilidade; ensaios não-destrutivos 


\section{INTRODUCCIÓN}

En 2015 se produjo una cumbre mundial denominada Cumbre del Desarrollo Sostenible que sentó las bases de la denominada Agenda 2030. Esta agenda contiene los denominados Objetivos de Desarrollo Sostenible (ODS) que buscan tener un mundo más sostenible para el año 2030. Los ODS son 17 objetivos de diversa índole que abarcan desde poner fin a la pobreza (ODS1) hasta la lucha contra el cambio climático (ODS13).

Respecto a este último objetivo hay que tener en cuenta que la lucha contra el cambio climático pasa por reducir la huella de carbono que afecta a nivel mundial. Esto implica la sustitución del modelo vigente de extracción de recursos naturales por un modelo de reutilización de residuos generados. La reutilización de residuos favorece la denominada economía circular, concepto económico que está íntimamente relacionado con la sostenibilidad y cuyo principal objetivo es que los productos, materiales y recursos se mantengan en el ciclo productivo el mayor tiempo posible y generando el menor número y cantidad posibles de residuos. Así, parte de los residuos generados pasan a ser productos que se pueden utilizar dentro del mismo sistema que los ha generado o en otra actividad distinta.

Una de las industrias que más negativamente ha impactado en la generación de gases de efecto invernadero es la industria de la construcción. Se considera que la producción del cemento portland es la responsable del 8-10\% de las emisiones totales de $\mathrm{CO}_{2}$ (Robayo Salazar et al, 2018). De esas emisiones de $\mathrm{CO}_{2}$, alrededor del $50 \%$ se producen debido a la calcinación de las materias primas (Benhelal et al, 2013). A lo largo de las últimas décadas se han considerado distintas alternativas para reducir el consumo de cemento portland mediante el uso de materiales suplementarios o mejoras en los procesos de producción del mismo (Juenger and Siddique, 2015; Li et al., 2019; Naqi and Jang, 2019). Estas alternativas no han conseguido reducir de manera suficientemente significativa la producción de $\mathrm{CO}_{2}$, es por ello necesario buscar alternativas que consigan sustituir en una medida sustancial el uso del cemento portland como material constructivo.

Los cementos de activación alcalina o materiales activados alcalinamente han emergido como materiales alternativos al cemento Portland produciendo, en muchos casos, una menor huella de carbono que este último (Assi et al, 2018). Además, frecuentemente los materiales que se utilizan para fabricar dichos cementos de activación alcalina son materiales residuales, con lo cual se está realizando varios beneficios: reducción de la huella de carbono, reducción económica al utilizar un residuo y contribución al desarrollo de la economía circular.

Algunos autores apuntan que la reducción de la huella de carbono en algunos casos es de hasta un $75 \%$ respecto al uso del cemento portland (Mellado et al, 2014, Yang et al 2013).

Entre los materiales más comúnmente empleados en la fabricación de los cementos de activación alcalina se encuentran la escoria de alto horno, la ceniza volante y el metacaolin, entre otros (Provis and Bernal, 2014; Shi et al, 2011). El uso de residuos cerámicos de diversos orígenes como materiales precursores para fabricación de matrices activadas alcalinamente se ha estudiado en los últimos años con diferentes resultados según el residuo empleado. Así se han empleado residuos de ladrillo rojo y gres porcelánico obteniendo resistencias mecánicas entre 22 y $41 \mathrm{MPa}$ para 7 días de curado a $65^{\circ} \mathrm{C}$ (Reig et al, 2013; Reig et a. 2014).

Específicamente, en varias investigaciones el uso de los residuos de la cerámica sanitaria (CS) ha demostrado la necesidad del aporte de una fuente de calcio para lograr que se produzca un endurecimiento de las matrices fabricadas con dicho residuo. La fuente de calcio puede ser mediante adición de hidróxido de calcio, cemento de aluminato de calcio o cemento portland, y la cantidad de estos últimos varía dependiendo del uso de una fuente de calcio u otra. Así el hidróxido de calcio no se puede añadir en cantidades superiores al $8 \%$ mientras que no existe limitación en el uso de cemento de aluminato de calcio (Reig et al, 2018). 
Las últimas investigaciones realizadas en este sentido abogan por la utilización de morteros mixtos de cerámica sanitaria con otros residuos, para lograr obtener matrices estables y con buenas resistencias mecánicas, curadas tanto a temperatura ambiente como curadas a $65^{\circ} \mathrm{C}(\mathrm{Cosa}$ et al; 2018; Cosa et al.; 2018). Los residuos empleados junto a la cerámica sanitaria en estas investigaciones han sido el residuo del catalizador de craqueo catalítico (FCC), la escoria de alto horno (ESC) y la ceniza volante (CV), obteniendo muy buenos resultados sobre todo cuando se combina su uso con el residuo del catalizador de craqueo catalítico (FCC) y la escoria.

El gran avance que les queda por recorrer a los materiales activados alcalinamente es estudiar su durabilidad. Tener un buen comportamiento mecánico no garantiza tener una buena durabilidad y este aspecto es muy importante para establecer una vida útil similar o superior a la alcanzada por matrices con cemento portland.

Uno de los ensayos importante para conocer la durabilidad de los materiales es el de realización de ciclos hielo-deshielo (H-D). Los matrices cementantes se someten a ciclos periódicos de hielo y deshielo durante un tiempo determinado y se estudia la degradación de las mismas.

Las investigaciones relacionadas con este ensayo en matrices de activación alcalina son muy diversas en cuanto a resultados obtenidos. Así, Pilhevar et al. estudiaron matrices de activación alcalina y de cemento Portland con y sin materiales encapsulados, sometidas a 28 ciclos H-D (Pilhevar et al., 2019). Los autores observaron que las matrices de activación alcalina tras 28 ciclos apenas mostraban variación en la resistencia a compresión, mientras que las muestras con cemento portland sufrían daños que hacían que disminuyera la resistencia.

Puertas et al. realizaron ensayos de H-D a matrices de activación con ceniza volante, con escoria y con mezcla de ambos y compararon los resultados con morteros de cemento Portland. (Puertas et al., 2003). Los morteros con cemento Portland y ceniza volante tras 50 ciclos H-D disminuían considerablemente su resistencia a flexotracción, mientras que los morteros con escoria y la mezcla de escoria y ceniza volante no solo no perdían resistencia mecánica tras los ciclos, sino que aumentaba el valor de las mismas.

Se ha encontrado una referencia donde un mortero de activación alcalina con ceniza volante se somete a 300 ciclos H-D reteniendo un $90 \%$ de la resistencia a compresión inicial que tenía antes de comenzar los ciclos (Sun and Wu, 2013).

Las técnicas de ensayo no destructivas son una herramienta muy útil para seguir el proceso de degradación de las matrices, sin necesidad de recurrir a un ensayo destructivo, como es la medida de las resistencias a flexotracción y a compresión. En este trabajo de investigación se han empleado dos técnicas no destructivas como son las técnicas NIRAS (Non-linear Impact Acoustic Spectrosccopy) y la velocidad de pulso ultrasónico. La técnica NIRAS detecta alteraciones en los materiales al monitorizar la variación de la frecuencia de resonancia en los modos de vibración cuando aumenta la energía de impacto a la que se somete una probeta de mortero. La evaluación del daño por la técnica del pulso ultrasónico se basa en la monitorización de la variación del pulso ultrasónico en función de los cambios que se van produciendo en la matriz cementante.

Estas técnicas ya se han empleado en estudios de durabilidad, como son los ataques por sulfatos (Carrión, 2017; Genovés 2017), demostrando su validez como técnicas de monitorización de daños sobre matrices cementicias.

En la presente comunicación se estudia el comportamiento de dos matrices de activación alcalina y una tradicional de cemento Portland, frente a los ensayos de ciclos de hielo-deshielo. Las matrices escogidas de activación alcalina se han seleccionado en base a estudios previos sobre estos materiales. Se ha caracterizado la resistencia mecánica de los morteros, antes de someterlos al ensayo de hielo-deshielo y después de 40 ciclos H-D. 


\section{PROCEDIMIENTO}

Se realizaron cinco amasadas de los tres tipos de morteros para analizar su evolución en los ensayos de H-D. Las tres matrices ensayadas fueron: un mortero de cemento portland CEM I-52.5 R con relación agua/cemento de 0.5; un mortero de activación alcalina utilizando un $100 \%$ de FCC como material precursor con relación agua/ $\mathrm{FCC}$ de 0.5 , y una disolución activadora con una relación molar $\mathrm{SiO}_{2} / \mathrm{Na}_{2} \mathrm{O}$ de 1.17 y una concentración de sodio de 7.5 molal; y un mortero de activación alcalina, utilizando como precursor una mezcla de $70 \%$ de CS con un 30\% de FCC con la misma dosificación del activador que la empleada en el otro mortero de activación. Los morteros con CS incorporan en su amasado un $4 \%$ de $\mathrm{Ca}(\mathrm{OH})_{2}$ para favorecer el proceso de geopolimerización. Todos los morteros presentan una relación árido/binder de $3 / 1$.

La cerámica sanitaria fue suministrada por la empresa Ideal Standard. Sse trata de piezas defectuosas del proceso de fabricación; dichas piezas se rompen y se pasan por una machacadora de mandíbulas (BB200 Restch). Tras su paso por la machacadora de mandíbulas, el tamaño de partícula es inferior a $2 \mathrm{~mm}$ y posteriormente se muele en un molino de jarras (Jars Turner Roller 1 Gabrielli) hasta obtener un polvo con un diámetro medio de $31.24 \mu \mathrm{m}$. El catalizador de craqueo catalítico fue suministrado por Omya Clariana S.A y viene molido de origen con un diámetro medio de $17.12 \mu \mathrm{m}$. El cemento Portland fue suministrado por la Lafarge Asland. Las composiciones químicas obtenidas por fluorescencia de rayos $\mathrm{X}$ se muestran en la tabla 1 .

Tabla 1. Composición química en porcentaje en óxidos del CEM I-52.5 R, CS y FCC

\begin{tabular}{|c|c|c|c|c|c|c|c|c|}
\hline & $\mathrm{Al}_{2} \mathrm{O}_{3}$ & $\mathrm{SiO}_{2}$ & $\mathrm{CaO}$ & $\mathrm{Fe}_{2} \mathrm{O}_{3}$ & $\mathrm{Na}_{2} \mathrm{O}$ & $\mathrm{K}_{2} \mathrm{O}$ & Otros & P.F \\
\hline CEM & 4.30 & 17.42 & 66.17 & 3.30 & 0.46 & 1.21 & 4.78 & 2.35 \\
\hline CS & 23.60 & 66.00 & 1.20 & 1.20 & 2.40 & 2.80 & 2.5 & 0.20 \\
\hline FCC & 49.26 & 47.76 & 0.11 & 0.6 & 0.31 & 0.02 & 1.43 & 0.51 \\
\hline
\end{tabular}

P.F pérdida al fuego

Las mezclas de activación alcalina se escogen basándose en los resultados obtenidos por Cosa et al. 2018. Los morteros de activación alcalina se curan durante 7 días en un baño térmico a $65^{\circ} \mathrm{C}$ mientras que los morteros de cemento se curan en cámara húmeda durante 28 días.

Una de las series amasadas se somete a ensayos mecánicos de rotura a flexotracción y a compresión según las indicaciones establecidas en la norma UNE-EN 196-1. El resto de series se someten a ciclos H-D en un equipo Ineltec modelo CRHEL-125 que realiza los ciclos de manera automática. Una vez transcurrido los ciclos escogidos, se sacan las probetas de la cámara y se dejan secar a temperatura ambiente hasta que se pierde la humedad superficial de las mismas. En la figura 1 se muestra una imagen de la cámara hielo-deshielo empleada para realizar el ensayo.

Las condiciones de los ciclos $\mathrm{H}-\mathrm{D}$ son las siguientes: se tarda una hora en alcanzar $-10^{\circ} \mathrm{C}$, posteriormente permanece a $-10^{\circ} \mathrm{C}$ durante 5 horas y en la fase deshielo permanece 1 hora a $15^{\circ} \mathrm{C}$.

Se realizan medidas de ensayos no destructivos sobre 12 probetas de cada tipo de mortero (4 series de 3 probetas cada serie). El seguimiento de la evolución por técnicas no destructivas se realiza antes del ensayo y después de 5, 15, 25, 40 y 70 ciclos H-D.

A 40 ciclos H-D se decide ensayar mecánicamente una de las series para obtener los datos de resistencia a flexotracción y a compresión y comparar los datos con los obtenidos a tiempo cero antes de someterlos a los ciclos H-D. Con el resto de series, se continua con su estudio hasta los 70 ciclos. 


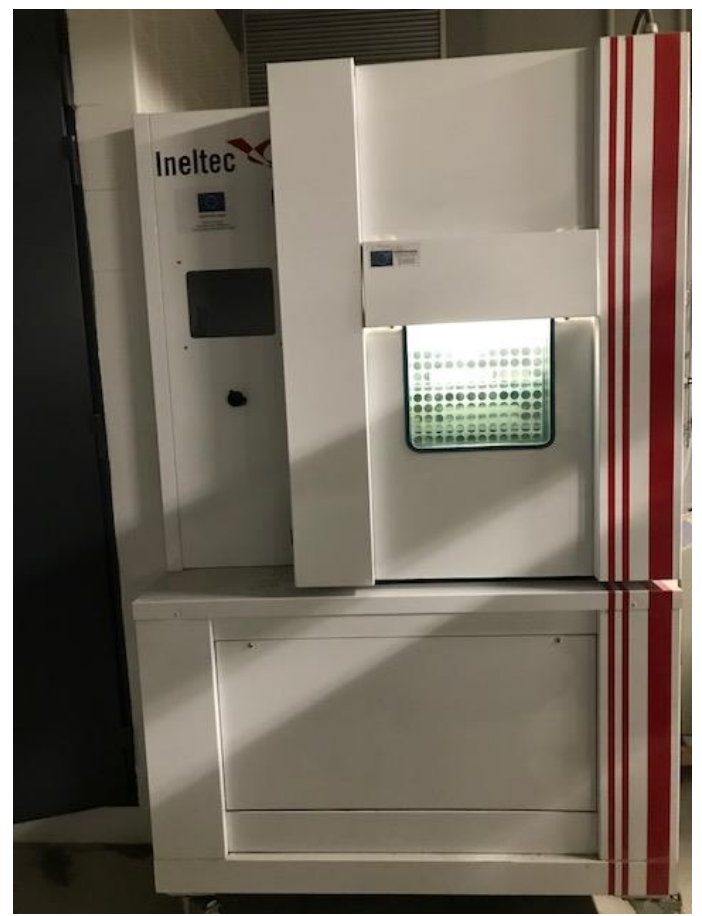

Figura 1. Cámara de hielo -deshielo empleada en el ensayo.

Los ensayos NIRAS necesitan diversa instrumentalización que a continuación se detalla brevemente. Se utiliza un soporte mecanizado donde está acoplado un martillo instrumentado, que se usa para generar el impacto que hace vibrar la probeta y que se utiliza para provocar diferentes niveles de impacto (figura 2a). A la probeta de ensayo se le coloca un captador acelerómetro piezoeléctrico (Piezotronics modelo 352A21) que capta la aceleración experimentada por la probeta. La señal captada por el acelerómetro se acondiciona y amplifica en un amplificador de señal (modelo PCB 482A18) para que sea adquirida y digitalizada por el osciloscopio. Las señales se captan en un osciloscopio (modelo MDO 314) y se genera un espectro de vibración. Con un software de control y procesamiento de los datos basados en Matlab se guardan los puntos del espectro.

El método de medida de US es el de transmisión directa. Los transductores (modelo General Electric K0,5SC) se acoplan a las caras opuestas de la probeta con ayuda de vaselina tal y como se muestra en la figura $2 \mathrm{~b}$. La transmisión se produce por un generador de señal programable modelo Agilent 33120A y la señal es adquirida y digitalizada por el mismo osciloscopio empleado en los ensayos NIRAS.
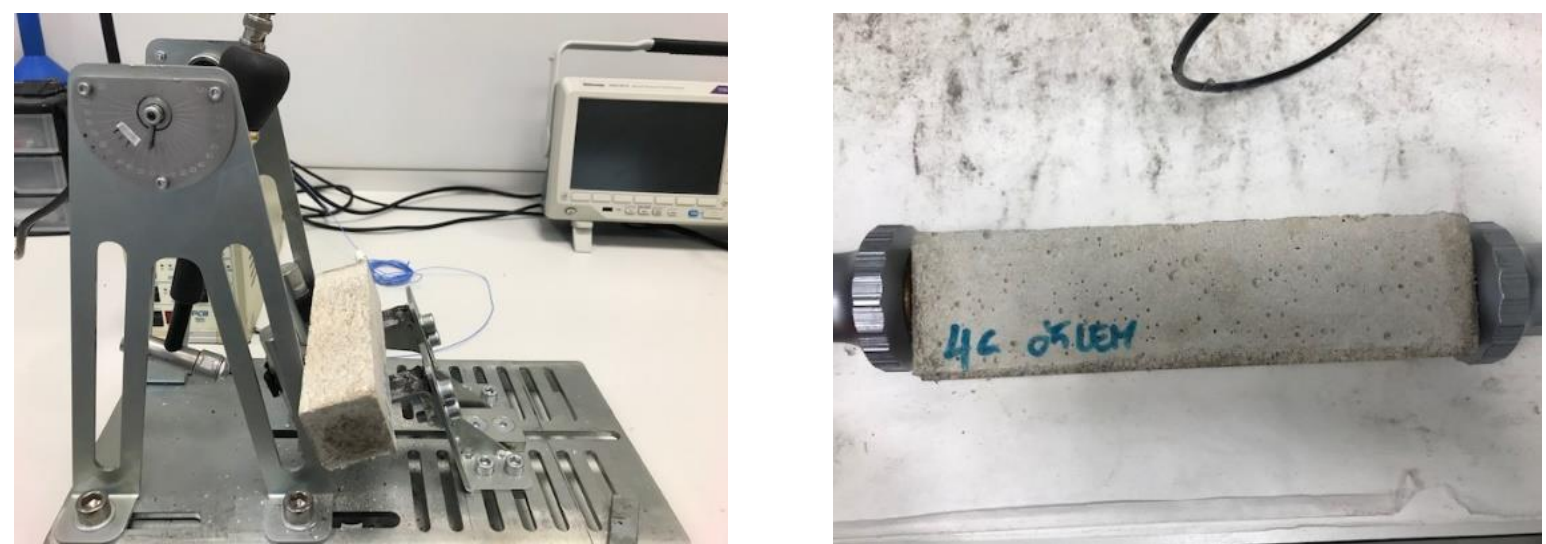

Figura 2. Ensayos no destructivos: a) Soporte y martillo para el ensayo NIRAS. b) Transductores acoplados a probetas para ensayo de US. 


\section{RESULTADOS}

A continuación, se presentan los resultados obtenidos tanto por las técnicas no destructivas como por los ensayos de resistencia a flexotracción y a compresión. En los ensayos de US se estudia dos parámetros: la atenuación de la señal ultrasónica y la velocidad de pulso ultrasónico; mientras que en los ensayos NIRAS se evalúan principalmente dos parámetros: el cambio en la frecuencia de resonancia lineal y el parámetro histerético.

\subsection{Ensayos de ultrasonidos: medida de la atenuación}

La atenuación presentada por los materiales analizados responde a la pérdida de intensidad de una onda mecánica que viaja a través de un medio. Mecanismos como el de reflexión, refracción o absorción hacen posible esta pérdida de intensidad de las señales ultrasónicas, siendo a su vez un indicador de las heterogeneidades de diferentes tamaños que se presentan en estos materiales.

Dado que, en las mezclas presentadas, el árido del mortero es igual para todos los casos, las diferencias existentes entre los tres materiales se deben principalmente a las diferencias entre la interfaz árido-pasta y la macro porosidad de las matrices de los morteros bajo estudio.

La figura 3 muestra los datos de atenuación de los tres tipos de morteros antes de comenzar los ciclos H-D, es decir, antes de sufrir ningún tipo de daño.

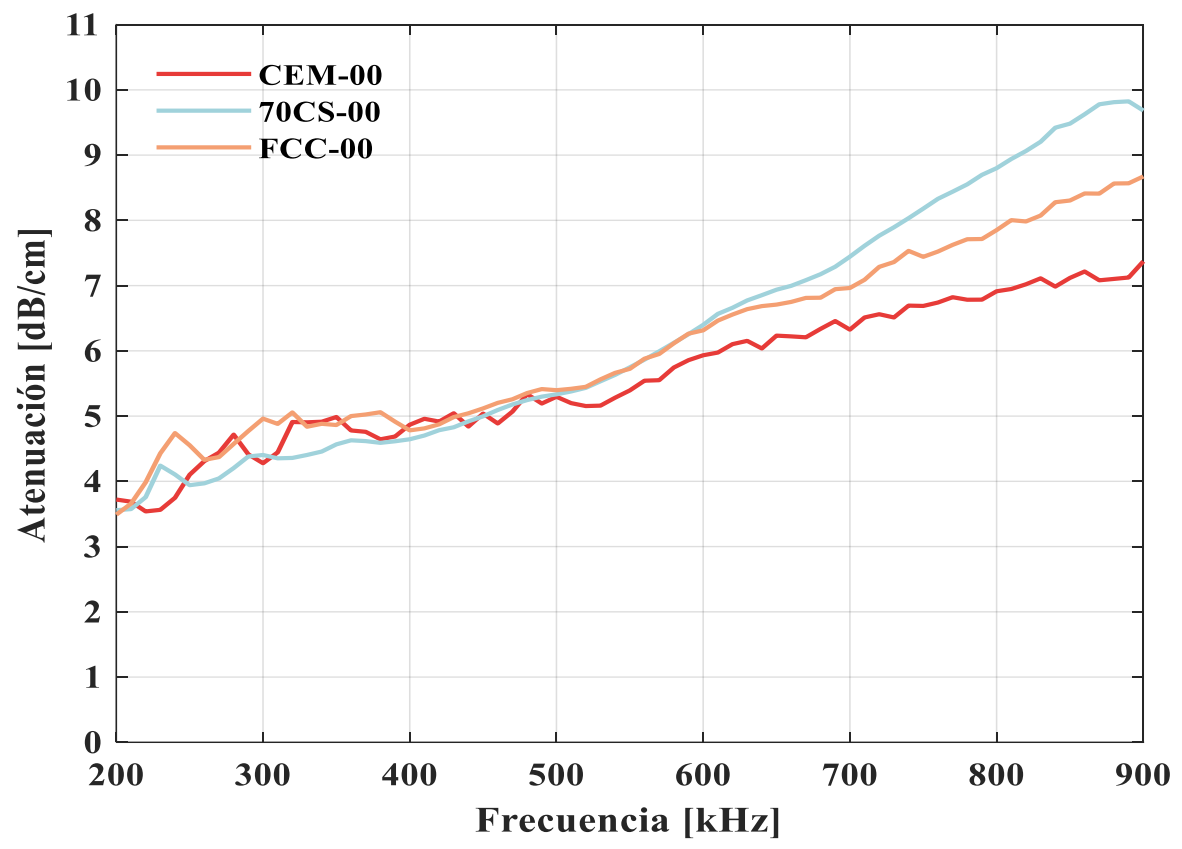

Figura 3. Resultados de atenuación previos al tratamiento de ciclos hielo-deshielo

Como se puede observar, los materiales antes del tratamiento H-D son diferentes, y se observan a partir de frecuencias superiores a los $500 \mathrm{~Hz}$. Es a partir de dicha frecuencia donde se aprecia que las curvas de cada serie se separan entre ellas. La serie cemento Portland (CEM) es la serie que menos atenúa y la serie con 70 CS-30FCC la de mayor atenuación. Ello puede ser debido a la mayor porosidad para la muestra de activación alcalina con CS o bien mayores defectos en la interfase árido/pasta de los áridos de mayor tamaño. 
Las figuras 4, 5 y 6 muestran los resultados de atenuación para las series de cemento Portland, FCC y 70CS-30FCC producidos a diferentes ciclos H-D. Importante destacar que no existen diferencias de atenuación significativas entre los ciclos realizados en ninguna serie y que los materiales de activación alcalina (FCC y 70CS-30FCC) no evolucionan de forma desfavorable durante el proceso de ciclos de hielo deshielo, teniendo diferencias relativas semejantes a las de la serie CEM. Esto sugiere que no se ha modificado de manera significativa la estructura interna de los especímenes, en especial la presencia de microfisuras, las cuales originarían un incremento de la atenuación con el número de ciclos.

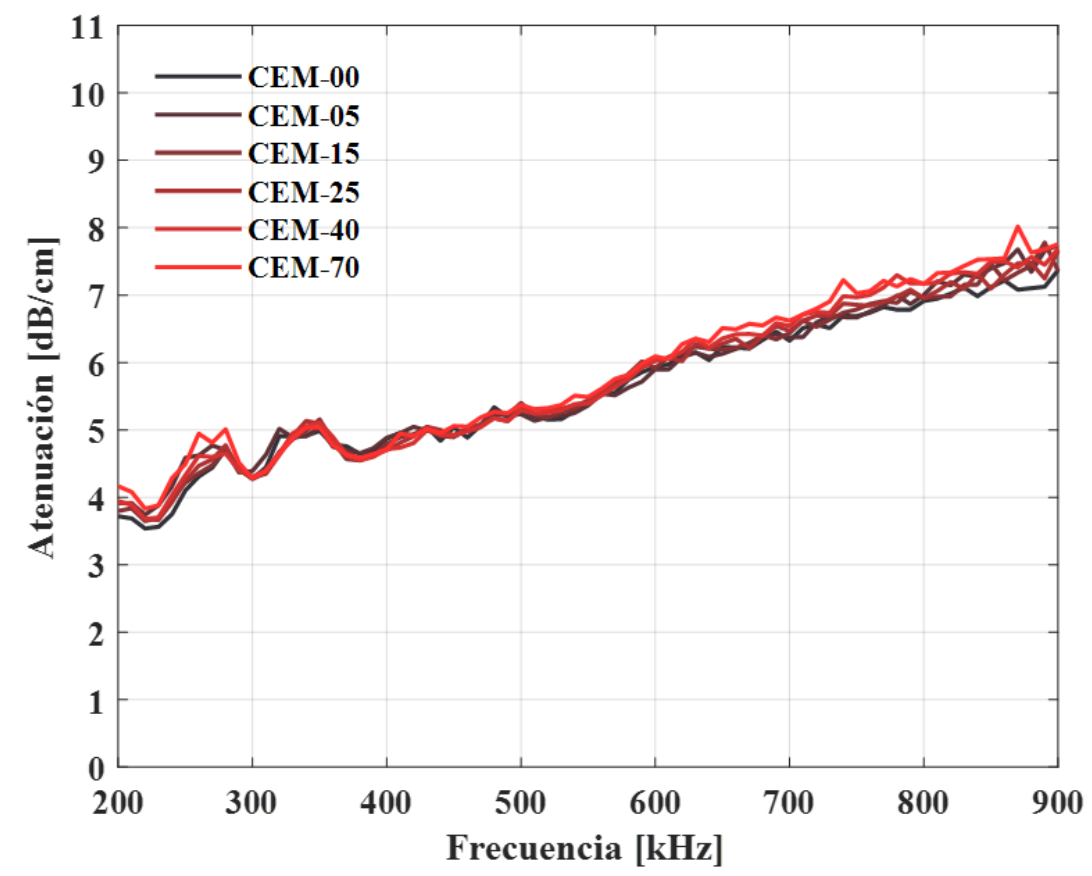

Figura 4. Curvas de atenuación para la serie de cemento Portland hasta 70 ciclos.

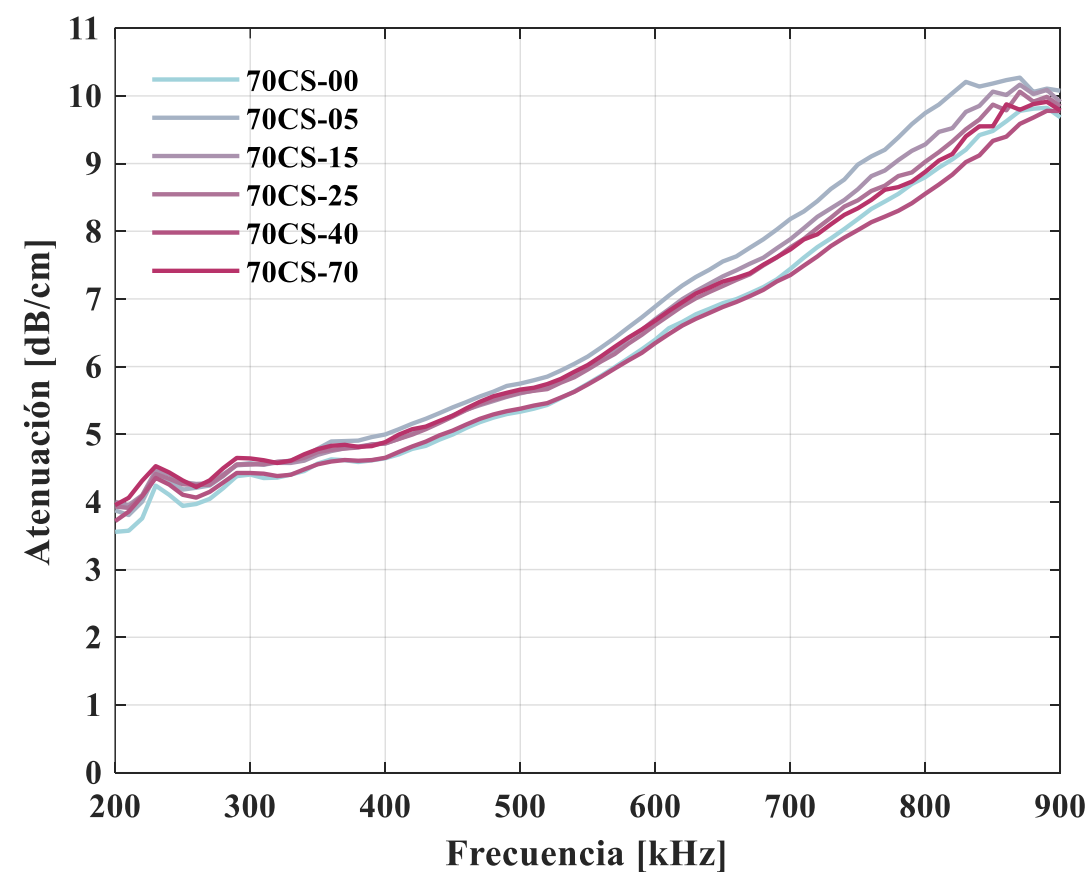

Figura 5. Curvas de atenuación para la serie de 70CS-30FCC hasta 70 ciclos. 


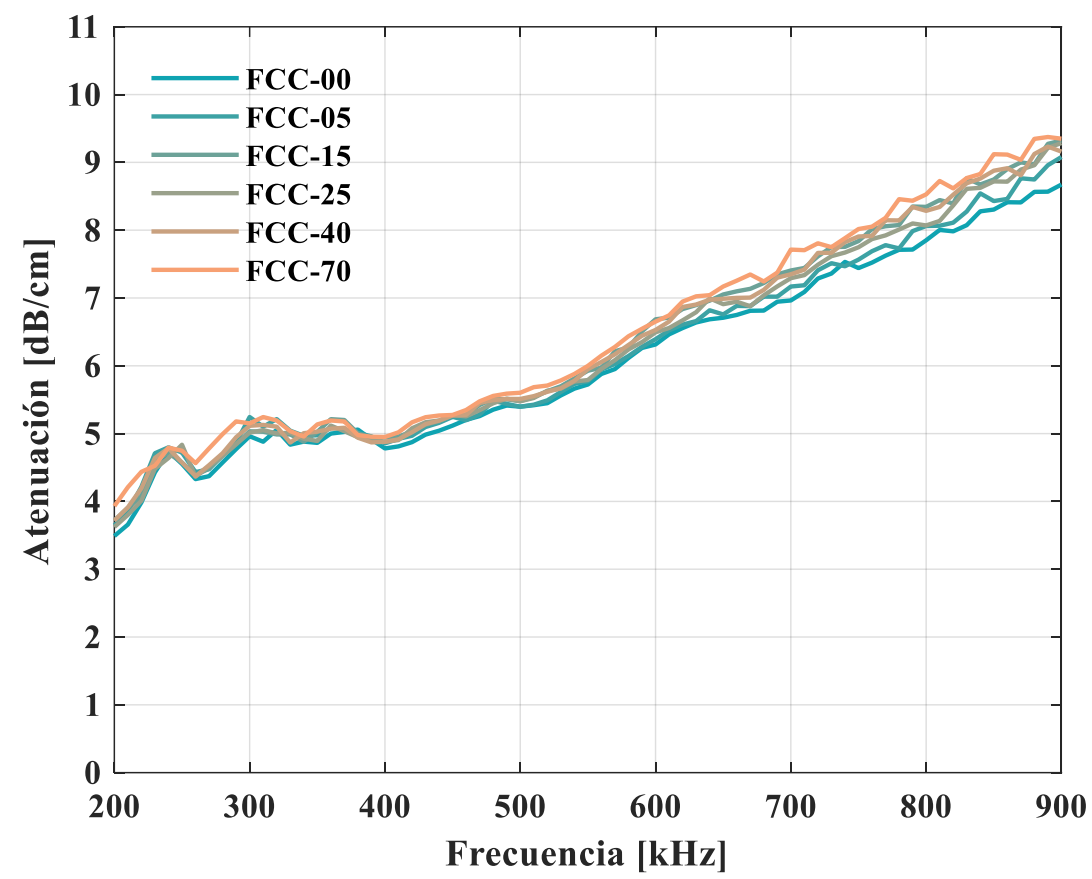

Figura 6. Curvas de atenuación para la serie de FCC hasta 70 ciclos.

\subsection{Velocidad de ultrasonidos}

La velocidad de una onda ultrasónica propagándose por un medio es proporcional a la rigidez de éste. Por lo tanto, el parámetro de velocidad ultrasónica es un dato importante para valorar el incremento o descenso de la rigidez de materiales en procesos de degradación o curado, en la comparación con materiales de diferente naturaleza.

En la figura 7 pueden apreciarse la velocidad de ultrasonidos para las series analizadas en función del número de ciclos de hielo-deshielo.

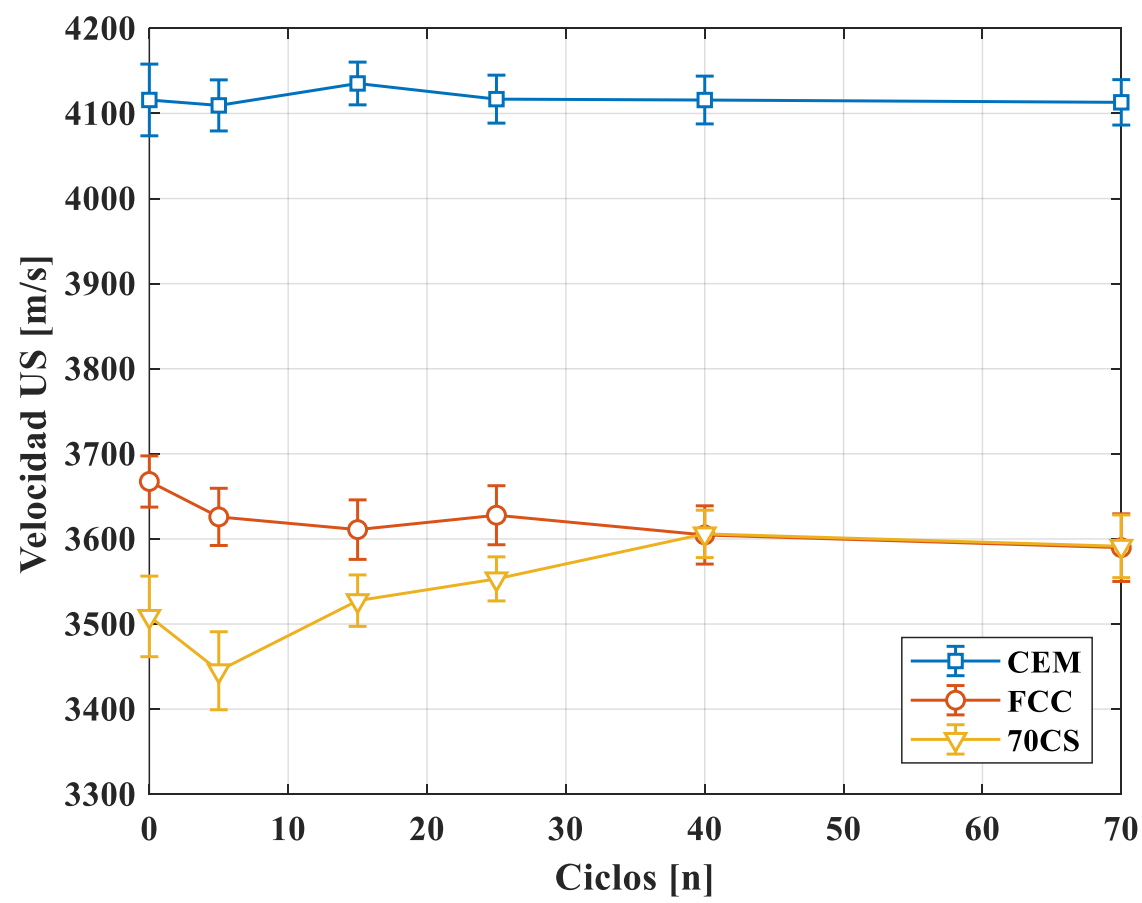

Figura 7. Velocidad de ultrasonido para los diferentes materiales analizados 
Tal y como se desprende de esta figura, la rigidez del mortero de cemento Portland es superior a la de la serie de FCC o 70CS-30FCC. Este parámetro sufre pequeñas fluctuaciones con cada serie de ciclos de hielo-deshielo pero no ofrece ninguna tendencia que indique que alguno de los materiales esté sufriendo un decremento de sus propiedades mecánicas (rigidez) con el tratamiento de daño. Sí se observa que, inicialmente, la rigidez de la mezcla 70CS-30FCC es inferior a la del otro sistema geopolimérico (FCC), pero que con los ciclos se produce un ligero incremento de la rigidez hasta prácticamente igualarse. Este comportamiento puede ser debido a que durante los procesos de hielodeshielo, se ha potenciado la reacción de geopolimerización. Ello sugiere que la CS tiene una velocidad de geopolimerización menor que FCC, y que requiere más tiempo para alcanzar su desarrollo óptimo.

\subsection{Ensayos de vibración: Medidas de frecuencia de resonancia lineal}

La frecuencia de resonancia de un elemento depende fundamentalmente de su masa, geometría y módulo dinámico. Dado que los elementos de geometría y masa se mantienen prácticamente constantes durante todo el experimental, el parámetro de frecuencia lineal es un indicador claro de la evolución del módulo dinámico y, por lo tanto, de la rigidez, a lo largo del experimental tal y como indica de igual forma la velocidad de ultrasonidos. La figura 8 muestra que no existe apenas variación para ninguno de los materiales a medida que avanza el número de ciclos, lo que corrobora una vez más que ninguno de los materiales parece haber sufrido daño significativo tras 70 ciclos H-D.

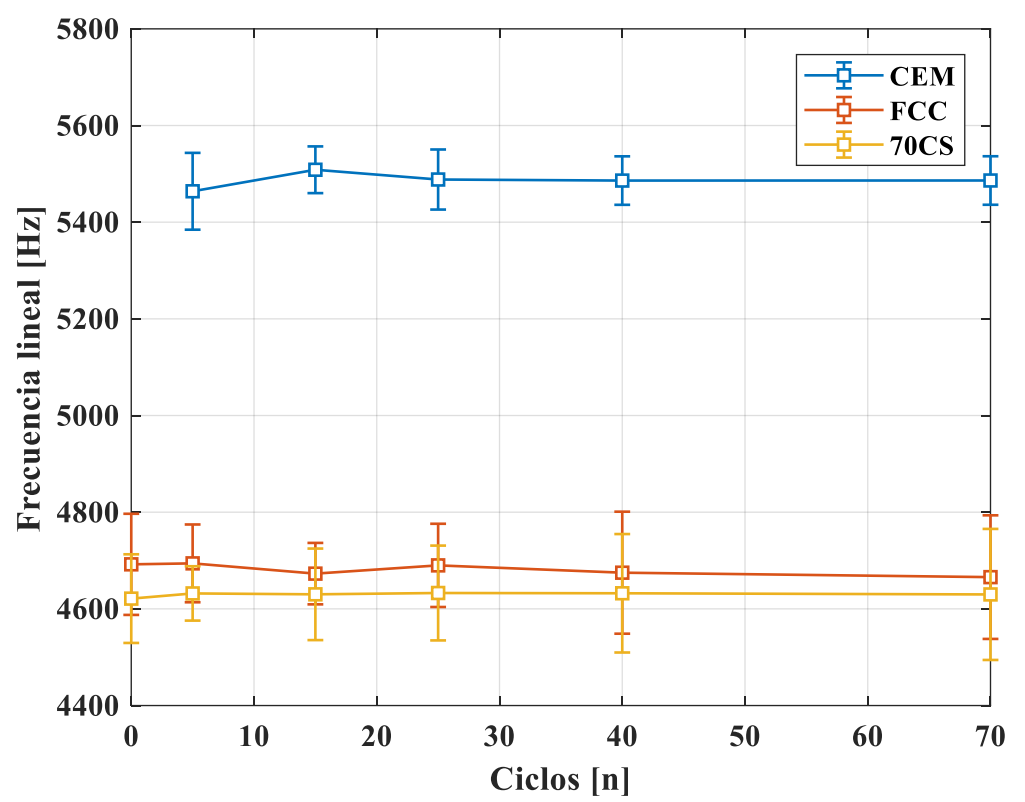

Figura 8. Ensayos de vibración: medidas de la frecuencia lineal para los diferentes materiales analizados y con diverso número de ciclos H-D.

\subsection{Ensayos de vibración: Cálculo del parámetro histerético}

El parámetro histerético extraído a partir de la técnica de NIRAS hace referencia al comportamiento no-lineal del material, al producirse un ablandamiento del módulo con el incremento de la intensidad de excitación mediante diferentes métodos (en este caso, por impacto). Esta no -linealidad es proporcional a la cantidad de heterogeneidades que presenta el material, tales como interfaces o fisuras. Por ello, es correcto afirmar que si un material sufre un proceso de microfisuración como es el caso de daño por hielo-deshielo, este efecto no-lineal se verá incrementado a medida que el material se deteriore. 
De la figura 9 puede concluirse que no hay cambios significativos en ninguna de las series, a medida que van realizándose ciclos de hielo-deshielo, por lo que cabe estimar que no se produce ningún daño apreciable tras 70 ciclos en ninguno de los materiales analizados.

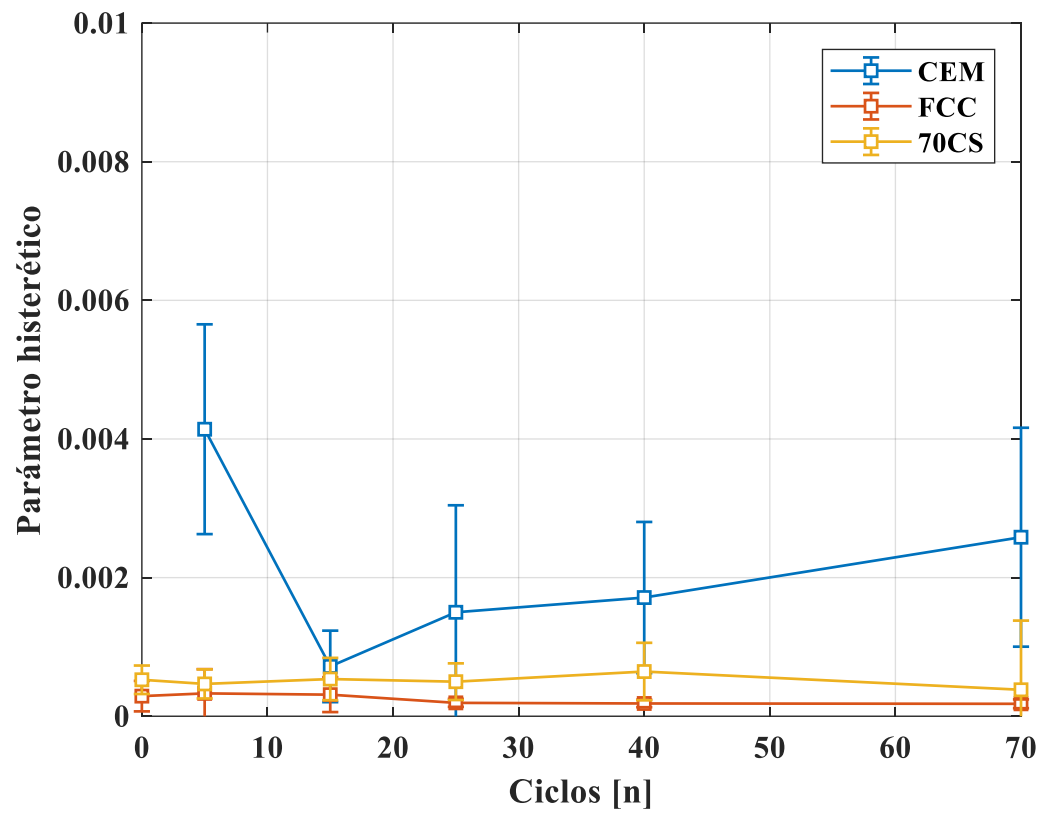

Figura 9. Parámetro histerético para los diferentes materiales analizados para diverso número de ciclos H-D.

\subsection{Ensayos destructivos: Medidas de resistencias mecánicas}

Se llevaron a cabo ensayos de resistencia a flexotracción y a compresión a una serie de las probetas antes de comenzar los ensayos H-D (punto inicial) y a otra serie de probetas tras realizarse 40 ciclos $\mathrm{H}$ D. Los resultados obtenidos se muestran en la figura 10a y 10b que representan los valores de resistencia flexotracción y a compresión respectivamente. Como se puede observar, la única serie que ha sufrido un descenso significativo al someterse a los ensayos H-D es la serie FCC y únicamente se ve disminuida ligeramente su resistencia a flexotracción, puesto que la resistencia a compresión no varía respecto al punto de partida.

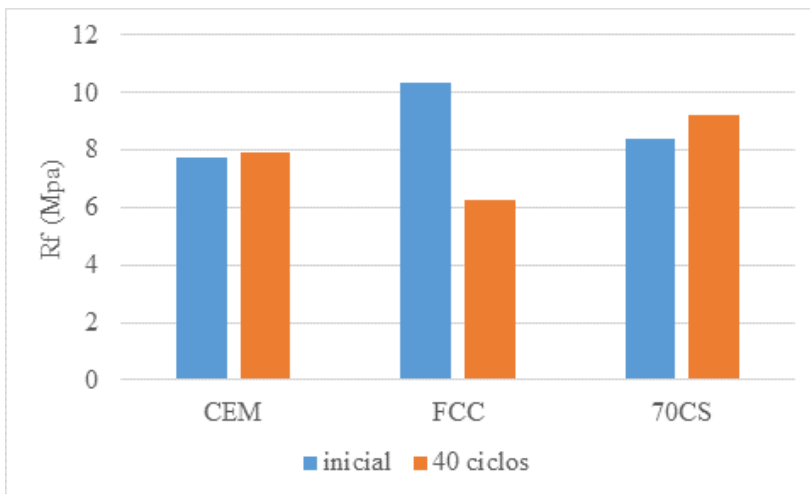

a)

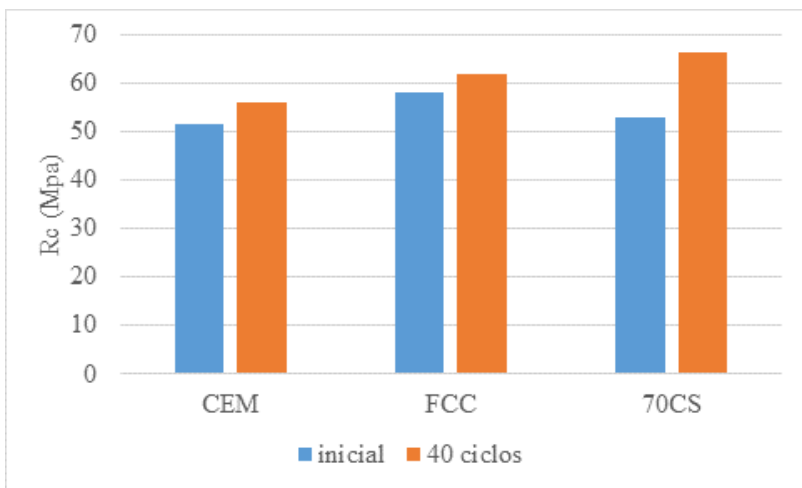

b)

Figura 10. Resistencias mecánicas para los diferentes materiales analizados antes de los ciclos H-D y después de 40 ciclos: a) Resistencia a flexotracción; b) Resistencia a compresión 
Los datos obtenidos son totalmente coherentes con los resultados obtenidos por los ensayos no destructivos; se ha concluido que no han mostrado tendencia a la baja. Se observa el mantenimiento, o incluso el ligero incremento de las resistencias mecánicas por parte de los distintos materiales ensayados. Puertas y col. ya comprobaron que algunas matrices de activación alcalina aumentan sus resistencias tras someterse a procesos de H-D, debido a que la reacción de activación alcalina puede continuar en ambientes de alta humedad y, por tanto, mejorar las prestaciones mecánicas (Puertas et al. 2003).

\section{CONCLUSIONES}

En el presente estudio se ha demostrado que se pueden fabricar matrices alternativas al cemento Portland con propiedades mecánicas y de durabilidad similares a este último. Además, estos materiales denominados de activación alcalina están fabricados utilizando residuos industriales como son la cerámica sanitaria y el catalizador de craqueo catalítico. Se han comparado sus prestaciones en ensayos de ciclos hielo-deshielo con un mortero de cemento Portland.

Los ensayos no destructivos de NIRAS y US apuntan a que los morteros ensayados no sufren alteraciones significativas tras someterse a 70 ciclos $\mathrm{H}-\mathrm{D}$, datos corroborados por ensayos destructivos de medida de resistencia mecánica a 40 ciclos H-D.

\section{AGRADECIMIENTOS}

Al Ministerio de Economía, Industria y Competitividad del Gobierno de España y a los fondos FEDER, por la concesión del proyecto coordinado BIA2017-87573-C2-1-P y BIA2014-55311-C2-1-P.

\section{REFERENCIAS}

Assi, L., Carter, K., Deaver, E, Anay, R., Ziehl, P. (2018), Sustainable concrete: Building a greener future. Journal of Cleaner Production. 198: 1641-1651. http://doi.org/10.1016/j.clepro.2018.07.123 Benhelal, E., Zahedi, G., Shamsaei, E., Bahadori, A. (2013), Global strategies and potentials to curb CO2 emissions in cement industry. Journal of Cleaner Production. 51: 142-161. http://dx.doi.org/10.1016/j.clepro.2018.07.123

Carrión, A., Genovés, J., Gosálbez, J., Miralles, R., Payá, J. (2017), Ultrasonic signal modality: A novel approach for concrete damage. Cement and Concrete Research. 101: 25-32. http://dx.doi.org/10.1016/j.cemconres.2017.08.011

Cosa, J., Soriano, L., Borrachero, M.V., Reig, L., Payá, J., Monzó, J.M. (2018), Influence of Addition of Fluid Catalytic Cracking Residue (FCC) and the $\mathrm{SiO}_{2}$ Concentration in Alkali-Activated Ceramic Sanitary-Ware (CSW). Minerals. 8 (123). doi:10.3390/min8040123

Cosa, J., Soriano, L., Borrachero, M.V., Reig, L., Payá, J., Monzó, J.M. (2018), The Compressive Strength and Microstructure of Alkali-Activated Binary Cements Developed by Combining Ceramic Sanitaryware with Fly Ash or Blast Furnace Slag. Minerals. 8 (337). doi:10.3390/min8080337

Genovés, J., Vargas, F., Gosálbez, J., Carrión, A., Borrachero, M.V., Payá, J. (2017), Ultrasonic and impact spectroscopy monitoring on internal sulphate attack of cement-based materials. Materials and Design. 125: 46-54. http://dx.doi.org/10.1016/j.matdes.2017.03.068

Juenger, M.C.G., Siddique, R. (2015), Recent advances in understanding the role of supplementary cementitious materials. Cement and Concrete Research. 78: 71-80. http://dx.doi.org/10.1016/j.cemconres.2015.03.018 
Li, C., Wu, M, Yao, W. (2019), Eco-efficient Cementitious System Consisting of Belite-Ye'elimiteFerrite Cement, Limestone Filler, and Silica Fume. ACS Sustainable Chemistry \& Engineering. 7: 7941-7950. DOI 10.1021/acssuschemeng.9b00702

Mellado, A., Catalán, C., Bouzon, N., Borrachero, M.V., Monzó, J.M., Payá, J. (2014), Carbon footprint of geopolymeric mortar: study of the contribution of the alkaline activating solution and assessment of an alternative route. RSC Advances. 4, 23846. DOI 10.1039/c4ra03375b

Naqi, A. Jang, J.G. (2019), Recent Progress in Green Cement Technology Utilizing Low-Carbon Emission Fuels and Raw Materials. Sustainability. 11, 537. doi 10.3390/su11020537

Pilehvar, S., Szczotok, A.M., Rodríguez, J.F., Valentini, L., Lanzón, M., Pamies, R, Kjǿniksen, A.L. (2019), Effect of freeze-thaw cycles on the mechanical behaviour of geopolymer concrete and Portland cement concrete containing micro-encapsulated phase change materials. Construction and Building Materials. 200: 94-103. http://doi.org/10.1016/j.conbuildmar.2018.12.057

Provis, J.L., Bernal, S.A (2014), Geopolymers and Related Alkali-Activated Materials. Annual Review of Materials Research. 44: 299-327 doi:10.1146/annurev-matsci-070813-113515

Puertas, F., Amat, T., Fernández-Jiménez, A, Vazquez, T. (2003), Mechanical and durable behaviour of alkaline cement mortars reinforced with polypropylene fibres. Cement and Concrete Research. 33: 2031-2036 doi:10.1016/S008-8846(03)00222-9

Reig, L., Tashima, M.M., Soriano, L., Borrachero, M.V., Monzó, J., Payá, J. (2013), Alkaline Activation of Ceramic Waste Materials. Waste Biomass Valor. 4: 729-736. DOI 10.1007/s12649-013-9197-z

Reig, L., Soriano, L., Borrachero, M.V., Monzó, J., Payá, J. (2014), Influence of the activator concentration and calcium hydroxide addition on the properties of alkali-activated porcelain stoneware. Construction and Building Materials. 63: 214-222 http://dx.doi.org/10.1016/j.conbuilmat.2014.04.023

Reig, L., Soriano, L., Tashima, M.M., Borrachero, M.V., Monzó, J., Payá, J. (2018), Influence of calcium additions on the compressive strength and microstructure of alkali-activated ceramics sanitary-ware. Journal of American Ceramic Society. 101: 3094-3104. doi 10.1111/jace.15436

Robayo-Salazar, R., Mejía-Arcila, J., Mejía de Gutierrez, R., Martínez, E. (2018), Life cycle assessment (LCA) of an alkali-activated binary concrete based on natural volcanic pozzolan: a comparative analysis to OPC concrete. Construction and Building Materials. 176: 103-11 http://dx.doi.org/10.1016/j.conbuilmat.2018.05.017

Shi, C., Fernández-Jiménez, A., Palomo, A. (2011), New cements for the $21^{\text {st }}$ century. The pursuit of an alternative to Portland Cement. Cement and Concrete Research. 41: 750-763. Doi:10.1016/j.cemconres.2011.03.016

Sun, P., Wu, H-C (2013). Chemical and freeze-thaw resistance of fly ash-based inorganic mortars. Fuel: 740-745. http://dx.doi.org/10.1016/j.fuel.2013.04.070.

Yang, K-H., Song, J-K, Song, H-I (2013). Assessment of CO2 reduction of alkali-activated concrete. Journal of Cleaner Production, 265-272. 